\section{Avaliação de medidas de controle de flebotomíneos no norte do Estado do Paraná, Brasil}

\author{
Evaluation of sandfly control measures in northern \\ Paraná State, Brazil
}

\author{
${ }_{1}^{1}$ Departamento de Análises \\ Clínicas, Universidade \\ Estadual de Maringá, \\ Maringá, Brasil. \\ $215 \underline{a}$ Regional de Saúde, \\ Secretaria de Saúde do \\ Paraná, Maringá, Brasil. \\ 3 Programa de Pós-graduação \\ em Análises Clínicas, \\ Universidade Estadual de \\ Maringá, Maringá, Brasil. \\ ${ }_{4}^{4}$ Programa de Pós-graduação \\ em Ciências da Saúde, \\ Universidade Estadual de \\ Maringá, Maringá, Brasil. \\ Correspondência \\ U. Teodoro \\ Departamento de Análises \\ Clínicas, Universidade \\ Estadual de Maringá. \\ Av. Colombo 5790, \\ Maringá, $P R$ \\ 87020-900, Brasil. \\ uteodoro@uem.br
}

\begin{abstract}
Sandfly captures from April 2001-September 2002 were compared to those from October 1996September 1997 and October 1998-April 2000 in order to evaluate control procedures conducted in Recanto Marista in the county of Doutor Camargo, Paraná State, Brazil. Sandflies were captured with Falcão traps twice a month, both in the domiciles and henhouses, from 10 PM to 2 AM. In 2001-2002, 199,821 sandflies were captured, with an hourly average of 1,625.5 insects; average captures in 1996-1997 and 1997-1998 were 1,641.9 and 806.7, respectively. Nyssomyia neivai was the most abundant species (90.4\%) in all habitats. This species, together N. whitmani, Migonemyia migonei, and Pintomyia fischeri, accounted for $99.9 \%$ of all the specimens captured. The total hourly average sandfly captures increased, but $85 \%$ of these insects were captured in henhouses built with the purpose of attracting sandfly specimens, while the density decreased in other environments, especially intradomiciliary.

Cutaneous Leishmaniasis; Vector Control; Psychodidae
\end{abstract}

\author{
Ueslei Teodoro 1 \\ Demilson Rodrigues dos Santos 2 \\ Ademar Rodrigues dos Santos ${ }^{2}$ \\ Otílio de Oliveira ${ }^{2}$ \\ Luís Paschoal Poiani 2 \\ João Balduíno Kühl 1 \\ Maria Valdrinez Campana Lonardoni 1 \\ Thaís Gomes Verzignassi Silveira 1 \\ Wuelton Marcelo Monteiro ${ }^{3}$ \\ Herintha Coeto Neitzke 4
}

\section{Introdução}

A leishmaniose tegumentar americana tem sido notificada em todos os estados do Brasil, com 605.062 casos relatados de 1980 a 20051 . Nesse período registraram-se 13.206 casos da doença no Estado do Paraná, principalmente no norte e oeste, correspondendo a $98,7 \%$ do total do Sul do Brasil 1,2.

Numerosos trabalhos de controle de flebotomíneos têm dado um enfoque para o uso de inseticidas químicos em paredes de casas e anexos (abrigos de animais, silos) 3,4,5,6,7,8,9, em ambientes silvestres 10,11,12 e impregnados em mosquiteiros 13,14,15,16,17. Essas medidas apesar de atrativas não são permanentes 18 , além do que, a precariedade das habitações, a descontinuidade e/ou o uso em épocas inapropriadas e os riscos ambientais advindos da aplicação de inseticidas em ambientes silvestres, constituem fatores limitantes para o controle de flebotomíneos 15,19,20,21. Há diversos registros do insucesso de medidas de desinsetização utilizadas para o controle de flebotomíneos, especialmente no peridomicílio 6,8,17. Além disso, a desorganização de órgãos públicos de saúde e as trocas constantes de especialistas em controle de vetores por agentes de saúde têm gerado a carência de pessoal capacitado para a execução de trabalhos de controle 22,23.

Medidas alternativas baratas, práticas e que possam ser incorporadas no dia-a-dia das populações que vivem em áreas de risco, podem di- 
minuir a incidência da leishmaniose tegumentar americana 21,24. O que pressupõe que a prevenção e o controle das leishmanioses requerem estudos de avaliação da efetividade com componentes da promoção da saúde e participação imprescindível da comunidade em sua implementação, para assegurar sua sustentabilidade 25 . Esses têm sido os objetivos de estudos realizados no Estado do Paraná 26,27,28.

Avalia-se, pela segunda vez, a reorganização e limpeza do peridomicílio, a desinsetização de domicílios e de abrigos de animais domésticos e o uso de galinheiros como barreiras zooprofiláticas, como medidas para reduzir a população de flebotomíneos no Recanto Marista e a freqüência desses insetos no domicílio.

\section{Métodos}

O trabalho foi realizado no Recanto Marista, nas margens do rio Ivaí, numa área que comporta uma mata remanescente bastante alterada, no Município de Doutor Camargo, a 52o13' Longitude Oeste e 23033' Latitude Sul, na mesorregião noroeste do Paraná 27,29 (Figura 1).

Após a primeira investigação, realizada de outubro de 1996 a setembro de 1997, foram introduzidas medidas para impactar o ambiente, evitar a formação de criadouros de flebotomíneos e controlar as formas adultas ${ }^{29}$. As medidas empregadas foram: (i) limpeza de matéria orgânica acumulada no solo (folhas, frutos, resíduos agrícolas, fezes de animais domésticos e restos de alimentos a eles oferecidos etc.); (ii) drenagem do solo; (iii) corte de algumas árvores para evitar o sombreamento e manter o solo seco; (iv) construção de galinheiros como barreiras zooprofiláticas; (v) proibição da permanência de cães; (vi) desinsetização de todas as edificações (residências, silos e outras) e abrigos de animais domésticos (galinheiros, currais etc.). Uma nova investigação foi realizada, de outubro de 1998 a abril de 2000, para avaliar o impacto das medidas adotadas 27. Daí em diante, a maioria das medidas acima referidas continua a ser empregada para evitar a presença de flebotomíneos no domicílio.

No presente trabalho as coletas de flebotomíneos foram feitas com armadilhas luminosas de Falcão ${ }^{30}$, duas vezes ao mês, das 22 às 2 horas, de março de 2001 a setembro de 2002, num total de 152 horas em cada armadilha, exceto na 11, onde foram feitas apenas sete coletas de quatro horas, perfazendo um total de 28 horas. As armadilhas foram instaladas em diversos ecótopos conforme se discrimina em seguida:

- Armadilha 1: na varanda da residência na entrada do Recanto Marista (E1);
- Armadilha 2: dentro de uma pocilga acoplada a um galinheiro, nos quais quase sempre havia um suíno e vários frangos para abate; periodicamente o plantel de aves era renovado (E2);

- Armadilha R: atrás da residência de um trabalhador (R);

- Armadilha 3: no interior de um curral, com dois bezerros, que foi demolido e reconstruído próximo da residência $\mathrm{R}$, em julho de 2001, onde as coletas passaram a ser feitas (E3);

- Armadilha 4: em um anexo ao lado de uma residência onde, eventualmente, são alojados trabalhadores temporários (E4);

- Armadilha 5: num galinheiro com 5 a 10 galinhas, a uma distância de 15 metros da residência do administrador (E5);

- Armadilha 6: na varanda da residência do administrador (E6);

- Armadilha 7: num galinheiro, distante $38 \mathrm{me}$ tros da residência do administrador, com 20 a 25 frangos para abate (E7);

- Armadilha 8: na entrada principal de um alojamento, às margens do rio Ivaí; neste ecótopo não foram feitas coletas no período desta pesquisa, mas o ecótopo foi mantido nas tabelas para não criar confusão nas comparações com as coletas de períodos anteriores (E8);

- Armadilha 9: num galinheiro com 7 a 10 galinhas (E9);

- Armadilha 10: num galinheiro com 7 a 10 galinhas (E10);

- Armadilha 11: num galinheiro com 7 a 10 galinhas (E11).

Os flebotomíneos foram processados e identificados no laboratório de Parasitologia Básica da Universidade Estadual de Maringá. A nomenclatura das espécies segue Galati ${ }^{31}$.

\section{Resultados}

Na Tabela 1, verifica-se que no período 20012002 foram coletadas as seguintes espécies de flebotomíneos: Brumptomyia brumpti (Larrousse), Brumptomyia cunhai (Mangabeira), Expapillata firmatoi (Barretto, Martins \& Pellegrino), Evandromyia cortelezzii (Brèthes), Migonemyia migonei (França), Nyssomyia neivai (Pinto), Nyssomyia whitmani (Antunes \& Coutinho), Pintomyia fischeri (Pinto), Pintomyia monticola (Costa Lima), Pintomyia pessoai (Coutinho \& Barretto) e Psathyromyia shannoni (Dyar). Nesse período foram coletados 199.821 flebotomíneos. Coletaram-se 180.574 exemplares de $N$. neivai que, juntamente com as espécies $N$. whitmani, M. migonei e P. fischeri, somaram 199.612 exemplares de flebotomíneos. 
Figura 1

Distribuição das armadilhas de Falcão nos diversos ecótopos no Recanto Marista, Município de Doutor Camargo, Paraná, Brasil.
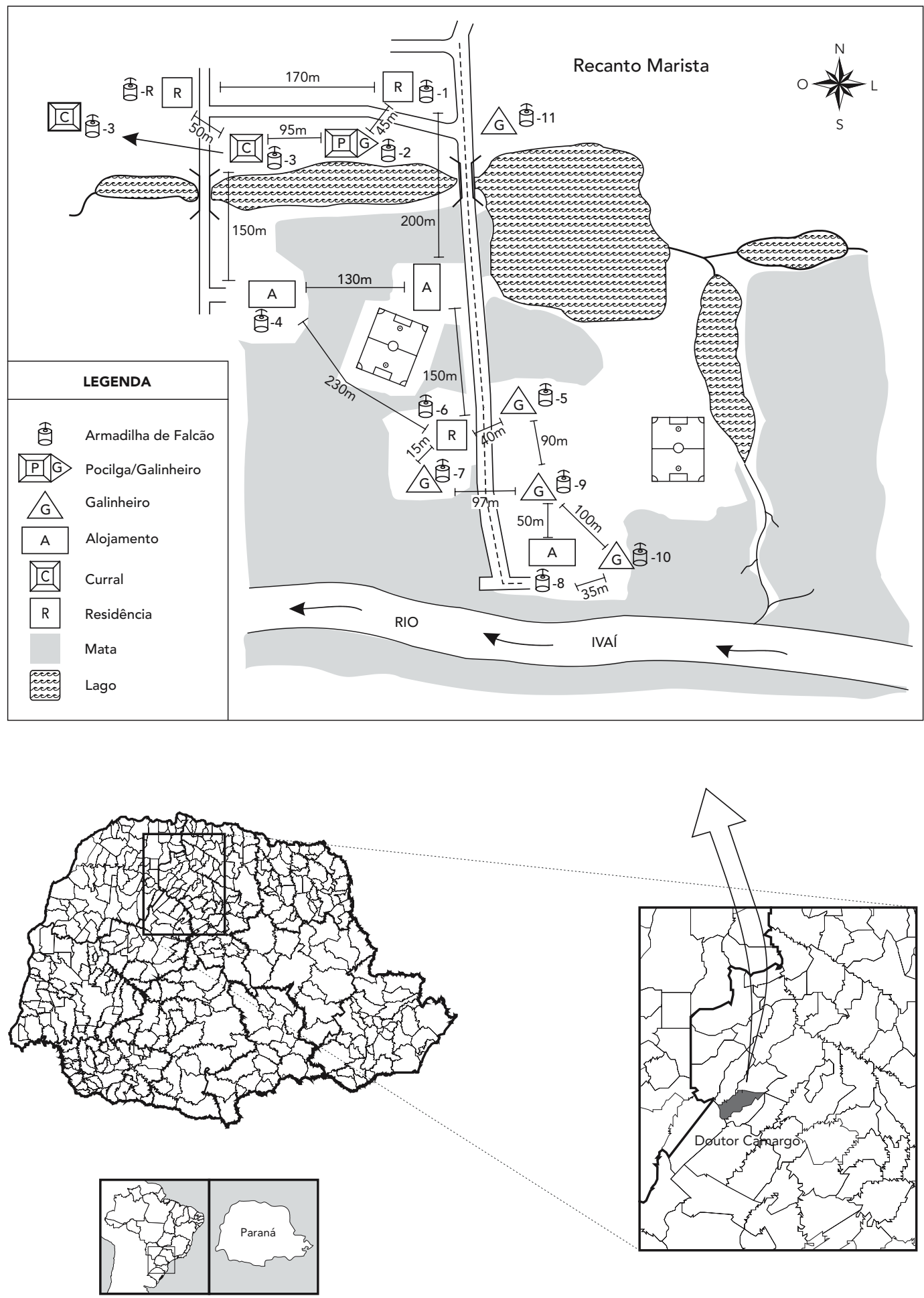
Flebotomíneos coletados no Recanto Marista, Município de Doutor Camargo, Paraná, Brasil, de março de 2001 a setembro de 2002.

\begin{tabular}{|c|c|c|c|c|c|c|c|c|c|c|c|c|c|c|}
\hline Espécie/Ecótopos & E1 & E2 & $\mathbf{R}$ & E3 & E4 & E5 & E6 & E7 & E8 & E9 & E10 & E11 & Total & $\%$ \\
\hline Nyssomyia neivai & 14.195 & 11.272 & 1.690 & 1.577 & 2.946 & 6.760 & 4.114 & 13.866 & - & 25.302 & 89.900 & 8.552 & 180.574 & 90,36 \\
\hline Nyssomyia whitmani & 3.974 & 3.380 & 318 & 167 & 531 & 171 & 166 & 370 & - & 1.189 & 4.140 & 2.585 & 16.991 & 8,50 \\
\hline Migonemyia migonei & 95 & 137 & 9 & 9 & 18 & 6 & 21 & 14 & - & 72 & 771 & 13 & 1.165 & 0,58 \\
\hline Pintomyia fischeri & 130 & 66 & - & - & 45 & 5 & 12 & 8 & - & 63 & 498 & 55 & 882 & 0,44 \\
\hline Pintomyia monticola & - & - & - & - & - & 1 & - & - & - & 1 & 13 & - & 15 & - \\
\hline Expapillata firmatoi & 1 & - & - & - & 1 & - & - & - & - & - & 2 & - & 4 & - \\
\hline Evandromyia cortelezzii & 3 & - & - & - & - & 1 & - & - & - & 2 & - & - & 6 & - \\
\hline Pintomyia pessoai & 12 & 14 & 1 & 2 & 1 & 3 & 2 & 5 & - & 15 & 53 & 23 & 132 & - \\
\hline Psathyromyia shannoni & - & - & 1 & - & - & - & - & 2 & - & 4 & 2 & 2 & 11 & - \\
\hline Brumptomyia brumpti & 1 & 1 & 2 & 1 & 1 & 1 & 2 & - & - & 5 & 22 & 2 & 37 & - \\
\hline Bumptomyia cunhai & 1 & - & - & - & - & - & - & - & - & - & 3 & - & 4 & - \\
\hline Total & 18.412 & 14.870 & 2.021 & 1.756 & 3.543 & 6.948 & 4.317 & 14.265 & - & 26.653 & 95.404 & 11.632 & 199.821 & - \\
\hline Horas de coletas & 152 & 152 & 152 & 152 & 152 & 152 & 152 & 152 & - & 152 & 152 & $28^{*}$ & - & - \\
\hline Média horária & 121,1 & 97,8 & 13,3 & 11,6 & 23,3 & 45,7 & 28,4 & 93,8 & - & 175,3 & 627,7 & 415,4 & $1.653,5$ & - \\
\hline
\end{tabular}

Localização das armadilhas: E1 e E6: varanda de residências; E2: dentro de uma pocilga acoplada a um galinheiro; R: fundo de uma residência; E3: interior de um curral; E4: anexo ao lado de uma residência; E5, E7, E9, E10 e E11: galinheiros; E8: alojamento.

Verifica-se na Tabela 2 que no ecótopo E10 coletou-se a maior média horária $(\mathrm{MH}=627,6)$ de flebotomíneos, seguido pelos ecótopos E11 $(\mathrm{MH}=415,4), \mathrm{E} 9(\mathrm{MH}=175,3)$ e $\mathrm{E} 1(\mathrm{MH}=121,1)$. Nos ecótopos E2, E5, E7, E9, E10 e E11, todos galinheiros, foram coletados $156.961(78,5 \%)$ insetos.

Verifica-se na Tabela 3 que os picos de flebotomíneos ocorreram nos meses de março, abril e novembro de 2001 e janeiro, fevereiro e março de 2002. Em 2001, nos diversos ecótopos, os picos ocoreram nos meses de março (E10), abril (E3), agosto (E4) e novembro (E9); em 2002, nos meses de janeiro (E6), fevereiro (R, E5), março (E1, E2, E7) e junho (E11).

\section{Discussão}

As espécies N. neivai, N. whitmani, M. migonei e $P$ fischeri representam $99,9 \%$ do total de flebotomíneos coletados. Não houve variação do número de espécies de flebotomíneos em relação às espécies coletadas em 1996/1997 e 1998/2000, enquanto as proporções das espécies mais freqüentes ( $N$. neivai, N. whitmani, M. migonei e $P$. fischeri) sofreram pequenas variações 27,29 . N. neivai predominou nas coletas atuais e nos períodos anteriores 27,29 , mostrando que esta espécie se adapta bem em ambientes antrópicos, inclusive em outros municípios no Paraná 32,33.
Destaca-se que a maioria dos flebotomíneos coletados foi $N$. neivai e que esta espécie pode estar envolvida na epidemiologia da leishmaniose tegumentar americana nesse recanto e em outras áreas onde tem sido coletada em grande número, no Estado do Paraná 32,33 .

Cabe lembrar que todos os galinheiros (E2, E5, E7, E9, E10, E11) foram construídos para atrair flebotomíneos, procurando diminuir a freqüência dos mesmos nos domicílios 27 . No período deste estudo foi feita apenas uma desinsetização dos galinheiros, do curral e de outras edificações com deltametrina (150mg/litro), em agosto de 2001.

A constatação de MH mais altas nos ecótopos E2, E5, E7, E9 e E10 do que em 1998/2000 27 foi surpreendente, pois durante o período de coletas verificou-se que muitas vezes as lâmpadas estavam queimadas, os galinheiros sujos e sem galinhas.

Os flebotomíneos coletados nos ecótopos E9 e E10 representaram $61,1 \%$ do total coletado, o que talvez possa ser explicado pelo fato destes galinheiros estarem localizados em áreas que têm o solo muito rico em matéria orgânica e com elevado teor de umidade, possibilitando a presença de criadouros destes insetos. Nas coletas realizadas em 1998-2000, nesses mesmos ecótopos, esses insetos representaram 58,7\% 27.

O ecótopo E11 foi construído em junho de 2002, com a finalidade de atrair os flebotomíneos que estavam sendo coletados em grande número 
Média horária (MH) de flebotomíneos coletados no Recanto Marista, Município de Doutor Camargo, Paraná, de outubro de 1996 a setembro de 1997*, de outubro de 1998 a abril de 2000 e de março de 2001 a setembro de 2002.

\begin{tabular}{|c|c|c|c|c|c|c|c|c|}
\hline \multirow[b]{2}{*}{ Ecótopo } & \multicolumn{2}{|c|}{$1996-1997$ * } & \multicolumn{3}{|c|}{$1998-2000$ ** } & \multicolumn{3}{|c|}{ 2001-2002 } \\
\hline & Total & $\mathrm{MH}$ & Ecótopo & Total & $\mathrm{MH}$ & Ecótopo & Total & MH \\
\hline M1 & 27.675 & 288,3 & E1 & 6.422 & 42,3 & E1 & 18.412 & 121,1 \\
\hline M2 & 3.332 & 34,7 & E2 & 5.155 & 33,9 & E2 & 14.870 & 97,8 \\
\hline- & - & - & - & - & - & $\mathrm{R}$ & 2.021 & 13,3 \\
\hline M3 & 78.710 & 819,8 & E3 & 4.376 & 28,8 & E3 & 1.756 & 11,6 \\
\hline M4 & 19.816 & 206,4 & E4 & 9.268 & 61,0 & E4 & 3.543 & 23,3 \\
\hline- & - & - & E5 & 5.295 & 34,8 & E5 & 6.948 & 45,7 \\
\hline M5 & 22.733 & 236,8 & E6 & 5.996 & 39,4 & E6 & 4.317 & 28,4 \\
\hline- & - & - & E7 & 10.054 & 66,1 & E7 & 14.265 & 93,8 \\
\hline M6 & 5.359 & 55,8 & E8 & 4.066 & 26,8 & E8 & - & - \\
\hline- & - & - & E9 & 17.401 & 114,5 & E9 & 26.653 & 175,3 \\
\hline- & - & - & E10 & 54.583 & 359,1 & E10 & 95.404 & 627,6 \\
\hline- & - & - & - & - & - & E11 & 11.632 & 415,4 \\
\hline Total & 157.625 & $1.641,9$ & Total & 122.616 & 806,7 & Total & 199.821 & $1.653,5$ \\
\hline
\end{tabular}

* Teodoro et al. 27,29. Somente os resultados em negrito são comparáveis. Horas de coletas = 152, exceto em E11 = 28; Nestes ecótopos foram instaladas armadilhas nos anos 1996-1997, 1998-2000 e 2001-2002.

Localização das armadilhas: E1 e E6: varanda de residências; E2: dentro de uma pocilga acoplada a um galinheiro; R: fundo de uma residência; E3: interior de um curral; E4: anexo ao lado de uma residência; E5, E7, E9, E10 e E11: galinheiros; E8: alojamento.

no ecótopo E1, representado por uma residência. A MH de flebotomíneos coletados no ecótopo E1 diminuiu de 126,0 para 103,0 após a construção do ecótopo E11. De junho a setembro de 2002, as MH no ecótopo E11 $(578,1 ; 476,5 ; 341,3 ; 58,0)$ foram sempre superiores às MH em E1 $(97,1 ; 289,7$; $19,7 ; 5,4)$. Nesse período, no ecótopo E11 as MH foram 6, 1,7, 17,3 e 10,4 vezes maiores do que as MH no E1.

Nos galinheiros acima referidos foram coletados $85,0 \%$ do total de flebotomíneos. Anteriormente, nos mesmos ecótopos, exceto o E11, foram coletados $75,4 \% 27$. O potencial de galinheiros 27,34,35 e abrigos de outros animais domésticos como barreiras zooprofiláticas tem sido constatado em diversas pesquisas, pois nestes ambientes a freqüência de flebotomíneos tem sido bem elevada 26,28,29,32,34,35,36. Outro fato que corrobora a importância de galinheiros como barreiras zoo-profiláticas é que o flebotomíneo infectado com Leishmania pode ter a infecção eliminada quando faz um segundo repasto em galinhas 37 .

As $\mathrm{MH}$ de flebotomíneos nos ecótopos $\mathrm{E} 4 \mathrm{e}$ E6 neste trabalho foram menores do que as $\mathrm{MH}$ em 1996-1997 e 1998-2000, mantendo baixa a densidade destes insetos, especialmente quando comparada com o número coletado no primeiro período. O primeiro ecótopo corresponde a um anexo ao lado de residência e o segundo a uma residência, indicando que as medidas de impacto no ambiente e a desinsetização das edificações que têm sido adotadas após o primeiro período de coletas devem ter contribuído para reduzir a densidade de flebotomíneos no domicílio, embora elas não venham sendo cumpridas de forma rigorosa 27.

A soma dos insetos coletados nos meses de janeiro, fevereiro, março e abril resultou em 136.670, representando $68,4 \%$ de um total de 199.821 flebotomíneos. Essa proporção é semelhante aos resultados observados anteriormente na mesma localidade 27 .

No mês de março de 2001 e 2002 foram coletadas as maiores proporções de flebotomíneos, respectivamente $31,9 \%$ e $14,4 \%$, perfazendo $46,3 \%$ (92.467 exemplares) do total de insetos coletados. Dos flebotomíneos coletados em março de 2001, 94,3\% (63.053 exemplares) foram coletados no E10 em apenas uma das coletas, representando $99,1 \%$ do total coletado neste mês. Anteriormente as maiores proporções de flebotomíneos coletados foram nos meses de janeiro e fevereiro. O que comprova uma vez mais que esses dípteros são mais freqüentes nos meses mais quentes e úmidos do ano 27. 
Sazonalidade do conjunto das espécies de flebotomíneos coletados no Recanto Marista, Município de Doutor Camargo, Paraná, Brasil, de março de 2001 a setembro de 2002

\begin{tabular}{|c|c|c|c|c|c|c|c|c|c|c|c|c|c|}
\hline \multirow[t]{2}{*}{ Mês } & \multicolumn{12}{|c|}{ Ecótopo } & \multirow[t]{2}{*}{ Total } \\
\hline & E1 & E2 & $\mathbf{R}$ & E3 & E4 & E5 & E6 & E7 & E8 & E9 & E10 & E11 & \\
\hline \multicolumn{14}{|l|}{2001} \\
\hline Mar & 398 & 48 & 39 & 228 & 100 & 787 & 91 & 818 & - & 1.103 & 60.032 & 0 & 63.644 \\
\hline Abr & 1.557 & 1.963 & 340 & 344 & 179 & 726 & 6 & 1.663 & - & 2.550 & 1.441 & 0 & 10.769 \\
\hline Mai & 114 & 93 & 0 & 6 & 24 & 0 & 48 & 4 & - & 0 & 9 & 0 & 298 \\
\hline Jun & 0 & 0 & 0 & 0 & 0 & 0 & 0 & 0 & - & 0 & 0 & 0 & 0 \\
\hline Jul & 843 & 271 & 78 & 40 & 339 & 907 & 249 & 464 & - & 3.811 & 1.349 & 0 & 8.351 \\
\hline Ago & 944 & 180 & 45 & 147 & 1.589 & 281 & 779 & 190 & - & 431 & 464 & 0 & 5.050 \\
\hline Set & 24 & 37 & 31 & 14 & 392 & 104 & 459 & 93 & - & 95 & 3.836 & 0 & 5.085 \\
\hline Out & 348 & 52 & 35 & 6 & 74 & 28 & 142 & 165 & - & 307 & 213 & 0 & 1.370 \\
\hline Nov & 933 & 384 & 21 & 163 & 232 & 3 & 626 & 1.552 & - & 4.879 & 2.789 & 0 & 11.582 \\
\hline Dez & 530 & 126 & 85 & 34 & 70 & 333 & 116 & 510 & - & 1.392 & 2.554 & 0 & 5.750 \\
\hline \multicolumn{14}{|l|}{2002} \\
\hline Jan & 1.784 & 1.098 & 78 & 68 & 15 & 1.084 & 912 & 1.203 & - & 3.556 & 2.133 & 0 & 11.931 \\
\hline Fev & 1.494 & 3.063 & 783 & 55 & 158 & 1.548 & 614 & 1.826 & - & 3.634 & 1.767 & 0 & 14.942 \\
\hline Mar & 5.001 & 6.182 & 238 & 251 & 105 & 239 & 66 & 2.706 & - & 652 & 13.383 & 0 & 28.823 \\
\hline Abr & 1.144 & 574 & 42 & 147 & 112 & 262 & 84 & 2.132 & - & 1.618 & 446 & 0 & 6.561 \\
\hline Mai & 3 & 0 & 1 & 4 & 0 & 17 & 2 & 160 & - & 207 & 65 & 0 & 459 \\
\hline Jun & 777 & 94 & 0 & 26 & 0 & 113 & 5 & 52 & - & 400 & 763 & 4.626 & 6.856 \\
\hline Jul & 2.317 & 441 & 188 & 149 & 54 & 134 & 59 & 157 & - & 437 & 298 & 3.812 & 8.046 \\
\hline Ago & 158 & 93 & 14 & 40 & 94 & 283 & 32 & 347 & - & 1.353 & 1.071 & 2.730 & 6.215 \\
\hline Set & 43 & 171 & 3 & 34 & 6 & 99 & 27 & 223 & - & 228 & 2.791 & 464 & 4.089 \\
\hline Total & 18.412 & 14.870 & 2.021 & 1.756 & 3.543 & 6.948 & 4.317 & 14.265 & - & 26.653 & 95.404 & 11.632 & 199.821 \\
\hline
\end{tabular}

Localização das armadilhas: E1 e E6: varanda de residências; E2: dentro de uma pocilga acoplada a um galinheiro; R: fundo de uma residência; E3: interior de um curral; E4: anexo ao lado de uma residência; E5, E7, E9, E10 e E11: galinheiros; E8: alojamento.

O crescimento da população de flebotomíneos no Recanto Marista pode ter ocorrido porque as medidas propostas para diminuir a densidade de flebotomíneos, principalmente a limpeza de matéria orgânica e as desinsetizações, não foram feitas rigorosamente. Contudo, a maioria dos flebotomíneos (85\%) foi coletada nos gali- nheiros construídos para atraí-los e diminuir sua densidade nos demais ambientes, especialmente no domicílio, mostrando a importância dos galinheiros como barreiras zooprofiláticas. Essas medidas de controle de flebotomíneos devem ser implantadas e avaliadas em outras áreas endêmicas de leishmaniose tegumentar americana. 


\section{Resumo}

Comparam-se os resultados de coletas de flebotomineos de abril de 2001 a setembro de 2002 com os resultados de coletas de outubro de 1996 a setembro de 1997 e de outubro de 1998 a abril de 2000, para avaliar as medidas empregadas para diminuir a densidade destes insetos, no Recanto Marista, Município de Doutor Camargo, Estado do Paraná, Brasil. As coletas de flebotomíneos foram feitas com armadilhas do tipo Falcão, em domicílios e galinheiros, das 22 às 2 horas, duas vezes ao mês. Em 2001/2002 coletaram-se 199.821 flebotomíneos, com média horária de 1.625,5 insetos; em 1996/1997 e 1997/1998, estas médias foram 1.641,9e 806,7 , respectivamente. Nyssomyia neivai predominou $(90,4 \%)$ em todos os ecótopos. Esta espécie, juntamente $\operatorname{com} \mathrm{N}$. whitmani, Migonemyia migonei $e$ Pintomyia fischeri representaram 99,9\% do total coletado. A média horária do total de flebotomíneos aumentou, contudo, 85\% destes insetos foram coletados nos galinheiros construídos com a finalidade de atraí-los, diminuindo a densidade dos mesmos nos demais ambientes, especialmente no domicílio.

Leishmaniose Americana; Controle de Vetores; Psychodidae

\section{Referências}

1. Ministério da Saúde. Leishmaniose tegumentar americana - distribuição de casos confirmados de LTA de 1980 a 2005. http://dtr2001.saude.gov. br/svs/epi/situacao_doencas/planilhas_doencas. htm (acessado em 12/Dez/2006).

2. Lima AP, Minelli L, Comunello E, Teodoro U. Distribuição da leishmaniose tegumentar por imagens de sensoriamento remoto orbital, no Estado do Paraná, Sul do Brasil. An Bras Dermatol 2002; 77:681-92.

3. Morsy TA, Aboul Ela RG, El Gozany BMR, Salama MMM, Raghed DA. Residual effect of four insecticides applied for indoor control of Phlebotomus papatasi (Scopoli). J Egypt Soc Parasitol 1993; 23:485-92.

4. Kelly DW, Mustafa Z, Dye C. Differential application of lambdacyhalothrin to control the sandfly Lutzomyia longipalpis. Med Vet Entomol 1997; 11:23-4.

5. Le Pont F, Padilla JM, Desjeux P, Richard A, Mouchet J. Impact de pulvérisations de deltraméthrine dans un foyer de leishmaniose de Bolivie. Ann Soc Belg Med Trop 1989; 69:223-32.

\section{Colaboradores}

U. Teodoro orientou a realização do trabalho e a elaboração do manuscrito. D. R. Santos contribuiu com a coleta, identificação dos flebotomíneos e elaboração do manuscrito. A. R. Santos, O. Oliveira e L. P. Poiani contribuíram com a coleta e identificação dos flebotomíneos. J. B. Kühl contribuiu com a identificação dos flebotomíneos. M. V. C. Lonardoni e T. G. V. Silveira contribuíram com a elaboração do manuscrito. W. M. Monteiro e H. C. Neitzke contribuíram com a identificação dos flebotomíneos e elaboração do manuscrito.

\section{Agradecimentos}

Ao Conselho Nacional de Desenvolvimento Científico e Tecnológico (CNPq) e à Fundação Araucária pelo apoio financeiro (Processo 40.0227/1999-1). Agradecemos também ao Colégio Marista de Maringá pelo apoio logístico.
6. Davies CR, Llanos-Cuentas EA, Campos P, Monge J, Leon E, Canales J. Spraying houses in the Peruvian Andes with lambda-cyhalothrin protects residents against cutaneous leishmaniasis. Trans R Soc Trop Med Hyg 2000; 94:631-6.

7. Falcão $A L$, Falcão $A R$, Pinto CT, Gontijo CMF, Falqueto A. Effect of deltamethrin spraying on the sandfly populations in a focus of American cutaneous leishmaniasis. Mem Inst Oswaldo Cruz 1991; 86:399-404.

8. Marcondes CB, Nascimento JA. Avaliação da eficiência de dentametrina (K-Othrine CE) no controle de Lutzomyia longipalpis (Diptera: Psychodidae), no Município de Santa Rita, Paraíba, Brasil. Rev Soc Bras Med Trop 1993; 26:15-8.

9. Teodoro U, Galati EAB, Kühl JB, Lozovei AL, Barbosa OC. Controle de flebotomíneos com DDT, em área endêmica de leishmaniose tegumentar no Estado do Paraná, sul do Brasil. Braz Arch Biol Technol 1998; 41:359-64.

10. Floch H. Sur la prophylaxie de la leishmaniose forestiére americaine. Riv Malariol 1957; 36:243-53. 
11. Ready PD, Arias JR, Freitas RA. A pilot study to control Lutzomyia umbratilis (Diptera: Psychodidae), the major vector of Leishmania braziliensis guyanensis in a peri-urban rainforest of Manaus, Amazonas State, Brazil. Mem Inst Oswaldo Cruz 1985; 80:27-36.

12. Perich MJ, Hoch AL, Rizzo N, Rowton ED. Insecticide barrier spraying for the control os sandfly vectors os cutaneous leishmaniasis in rural Guatemala. Am J Trop Med Hyg 1995; 52:485-8.

13. Chavasse DC, Reed C, Attawell K. Insecticidetreated net projects: a handbook for managers. London: Malaria Consortium, London School of Hygiene \& Tropical Medicine; 1999.

14. Desjeux P. Pyrethroid ampregnated bed nets: an alternative vector control approach for leishmaniasis. In: Proceedings of 13th European SOVE Meeting. Caglar SS, editor. Belek: Society for Vector Ecology; 2000. p. 152.

15. Feliciangeli MD, Maroli M, Wheeler A, Townson H, Ward R, Maignon R. Sandfly control trial with deltamethrin impregnated curtains in El Ingenio, Miranda State, Venezuela. Bol Dir Malar San Amb 1995; 35:12732.

16. Kroeger A, Avila EV, Morison L. Insecticide impregnated curtains to control domestic transmission of cutaneous leishmaniasis in Venezuela: cluster randomised trial. BMJ 2002; 325:810-3.

17. Alexander B, Usma MC, Cadena H, Quesada BL, Solarte Y, Roa W, et al. Evaluation of deltamethrinimpregnated bednets and curtains against phlebotomine sanflies in Valle del Cauca, Colombia. Med Vet Entomol 1995; 9:279-83.

18. Davies CR, Llanos-Cuentas A, Canales J, Leon E, Alvarez E, Monge J, et al. The fall and rise of Andean cutaneous leishmaniasis: transient impact of the DDT campaign in Peru. Trans R Soc Trop Med Hyg 1994; 88:389-93.

19. Alencar JE. Profilaxia do calazar no Ceará, Brasil. Rev Inst Med Trop São Paulo 1961; 3:175-80.

20. Alencar JE. Expansão do calazar no Brasil. Ceará Med 1983; 5:86-120.

21. Alexander B, Maroli M. Control of phlebotomine. Med Vet Entomol 2003; 17:1-18.

22. Sen Gupta PC. Return of kala-azar. J Indian Med Ass 1975; 65:89-90.

23. Townson H, Nathan NB, Zaim M, Guillet P, Manga $\mathrm{L}$, Bos R, et al. Exploiting the potential of vector control for disease prevention. Bull World Health Organ 2005; 83:942-7.

24. Campbell-Lendrum D, Dujardin JP, Martinez E, Feliciangeli MD, Perez JE, Silans LNMP, et al. Domestic and peridomestic transmission of American cutaneous leishmaniasis: changing epidemiological patterns present new control opportunities. Mem Inst Oswaldo Cruz 2001; 96:159-62.

25. Ampuero J, Urdaneta M, Macedo VO. Fatores de riesgo para la transmisíon de leishmaniasis cutánea en niños de 0 a 5 años en un área endémica de Leishmania (Viannia) braziliensis. Cad Saúde Pública 2005; 21:161-70.
26. Teodoro U, Kühl JB, Thomaz-SoccolV, Barbosa OC Ferreira MEMC, Lozovei AL, et al. Environmental sanitation and peridomiciliar organization as auxiliary practices for the control of phlebotomines in Paraná State, Southern Brazil. Braz Arch Biol Tecnol 1999; 42:307-14.

27. Teodoro U, Silveira TGV, Santos DR, Santos ES, Santos AR, Oliveira O, et al. Influência da reorganização, da limpeza do peridomicílio e da desinsetização de edificações na densidade populacional de flebotomíneos no Município de Doutor Camargo, Estado do Paraná, Brasil. Cad Saúde Pública 2003; 19:1801-13.

28. Teodoro U, Thomaz-Soccol V, Kühl JB, Santos DR, Santos ES, Santos AR, et al. Reorganization and cleaness of peridomiciliar area to control sand flies (Diptera, Pschodidae, Phlebotominae) in South Brazil. Braz Arch Biol Tecnol 2004; 47:205-12.

29. Teodoro U, Silveira TGV, Santos DR, Santos ES, Santos AR, Oliveira O, et al. Freqüência da fauna de flebotomíneos no domicílio e em abrigos de animais domésticos no peridomicílio, nos municípios de Cianorte e Doutor Camargo - Estado do Paraná - Brasil. Rev Patol Trop 2001; 30:209-33.

30. Falcão AR. Um novo modelo de armadilha luminosa de sucção para pequenos insetos. Mem Inst Oswaldo Cruz 1981; 76:303-5.

31. Galati EAB. Morfologia e taxonomia. In: Rangel EF, Lainson R, organizadores. Flebotomíneos do Brasil. Rio de Janeiro: Editora Fiocruz; 2003. p. 23-51.

32. Membrive NA, Rodrigues G, Membrive U, Monteiro WM, Neitzke HC, Lonardoni MVC, et al. Flebotomíneos de municípios do Norte do Estado do Paraná, Sul do Brasil. Entomol Vectores 2004; 11:673-80.

33. Teodoro U, Santos D, Santos AR, Oliveira O, Santos ES, Neitzke HC, et al. Avaliação de medidas de controle de flebotomíneos no Município de Lobato, Estado do Paraná, Sul do Brasil. Cad Saúde Pública 2006; 22:451-5.

34. Teodoro U, Kühl JB, Abbas M, Dias AC. Luz e aves como atrativos de flebotomíneos (Diptera, Psychodidae), no Sul do Brasil. Rev Bras Entomol 2001; 45:167-72.

35. Teodoro U. Características ecológicas de flebotomíneos (Díptera, Psychodidae) em habitats antrópicos, Município de Jussara, Paraná, Brasil. Rev Soc Bras Med Trop 1996; 29:625-6.

36. Teodoro U, Santos DR, Santos AR, Oliveira O, Poiani LP, Silva AM, et al. Informações preliminares sobre flebotomíneos do Norte do Paraná. Rev Saúde Pública 2006; 40:327-30.

37. Alexander B, Carvalho RL, McCallum H, Pereira MH. Role of domestic chicken (Gallus gallus) in the epidemiology of urban visceral leishmaniasis in Brazil. Emerg Infect Dis 2002; 8:1480-5.

Recebido em 06/Jul/2006

Versão final reapresentada em 19/Jan/2007

Aprovado em 07/Mai/2007 